\title{
Preferencia de forofito por los líquenes en el bosque chaqueño oriental
}

\author{
Preference of phorophyte by the lichens in the eastern Chaco forest
}

\author{
Cecilia Estrabou \\ Universidad Nacional de Córdoba, Facultad de Ciencias Exactas, Físicas y Naturales, \\ Centro de Ecología y Recursos Naturales Dr. Ricardo Luti, Av. Vélez Sarsfield 299, X5000JJC Córdoba, Argentina, \\ cecilia.estrabou@gmail.com
}

\begin{abstract}
SUMMARY
The epiphyte, corticolous lichen community depends on substrate to survive, they grow on trees being a non-timber resource. Many insects develop on it. The Chaco forest, a xerophytes environment, covers an extensive area in the north of Argentina with fine wood trees. The destruction of the forest jeopardizes the lichen community. The lichen community (life forms, structure and cover) is analyzed on the three most important phorophyte (dominant trees) in Chaco forest. Braun Blanquet techniques were used to obtain the data, an ordination technique (Principal Components Analysis), and Shannon index descriptive statistics to analyze them. 21 species are reported, the most favorable substrate was Zizyphus mistol with a total of 20 species. 16 species were registered on Prosopis pugionata, and 15 on Aspidosperma quebracho-blanco. The presence of lichen communities in the Chaco forest is discussed.
\end{abstract}

Key words: community, lichens, Chaco forest, diversity.

\section{RESUMEN}

Los líquenes epifitos y corticícolas dependen del sustrato para su supervivencia, crecen sobre árboles, son un recurso no maderero y sobre ellos se desarrollan gran cantidad de insectos. Una gran área del norte de la República Argentina es ocupada por el bosque chaqueño, con árboles de buena madera. La tala indiscriminada de este bosque pone en peligro su subsistencia y la de las especies liquénicas que viven en él. Se describió la comunidad liquénica del Chaco oriental, un ambiente xerofítico, sobre los tres forofitos (especies arbóreas dominantes) más favorables de ese bosque. Se utilizó la técnica de Braun Blanquet en la obtención de los datos, técnica de ordenación (análisis de componentes principales), índice de Shannon y estadística descriptiva para el análisis de los mismos. Se encontró un total de 21 especies, siendo el sustrato más favorable para el desarrollo de líquenes Zizyphus mistol, con un total de 20 especies. Sobre Prosopis pugionata se registraron 16 especies y 15 sobre Aspidosperma quebracho-blanco. Se discute la existencia de comunidades de líquenes presentes en el bosque chaqueño.

Palabras clave: comunidad, líquenes, bosque chaqueño, diversidad.

\section{INTRODUCCIÓN}

La planicie del Chaco ocupa la porción centro norte de Argentina con una superficie de alrededor de $500 \mathrm{mil}$ kilómetros cuadrados. La porción nororiental es el Chaco semiárido de la provincia de Córdoba, que se caracteriza por precipitaciones que oscilan entre 500 y $750 \mathrm{~mm}$ al año (Karlin et al. 1994). Fitogeográficamente, el paisaje original del Chaco consistía en manchones de bosque alternando con abras de pastizales originadas artificialmente. Los principales componentes arbóreos del bosque son Aspidosperma quebracho-blanco Schl., Acacia praecox Griseb., Zizyphus mistol Griseb. y Prosopis pugionata Burkart (Cabido y Zak 1999).

La disminución de la superficie del bosque en la actualidad se debe al avance de la frontera agropecuaria, en particular la siembra de soja (Glycine max (L.) Merr.). También el bosque chaqueño sufre un serio retroceso por la obtención de carbón y el uso domiciliario de la madera. Esto pone en peligro de pérdida a gran cantidad de especies asociadas al mismo, incluyendo los líquenes epifitos que se desarrollan sobre los árboles (Karlin et al. 1994). Se estima que la tasa de desmonte anual en las sierras es de 3,13\%, mientras que en la llanura es de 2,75\% (Zak et al. 2004). Desde el año 2001, durante el cual se comenzó este trabajo, hasta la actualidad, han desaparecido grandes superficies de bosque nativo, entre las que se encontraba uno de los sitios de muestreo. En el presente trabajo se describe la estructura de la comunidad liquénica del Chaco Oriental sobre tres especies arbóreas representativas de dicho bosque: A. quebrachoblanco, P. pugionata y Z. mistol. 
Los talos liquénicos presentan una amplia variedad de posibilidades de uso. En gran parte del mundo, incluido Argentina, los aborígenes los utilizaron en diversas artesanías y ritos mitomágicos (Llano 1951); también los usaron, costumbre que aún persiste, como recurso alimenticio, tintóreo y medicinal como en el tratamiento de la tuberculosis y del carcinoma de pulmón (Elix 1996). En la ciudad de Córdoba se ha corroborado la venta de talos liquénicos como producto de herboristería para la realización de infusiones. No obstante, es incipiente la línea de investigación sobre la reproducción, productividad y sustentabilidad de este grupo.

\section{MÉTODOS}

El área de estudio se ubica en la llanura del noreste de la provincia de Córdoba, a $30 \mathrm{~km}$ al norte de Sebastián Elcano, e incluye el área de reserva "La Candelaria". Presenta clima continental, cálido con temperatura media de $23{ }^{\circ} \mathrm{C}$ (Cabrera 1971). La región estudiada incluye vegetación dominada por $A$. quebracho-blanco y como especies secundarias a Z. mistol, Geoffroea decorticans (Gillies ex Hook. et Arn.) Burkart, P. alba Gris., P. nigra (Gris.) Hieronymus, Acacia spp. y Celtis spp. entre otras (Prado 1993).

Se trabajó sobre tres forofitos ya que la mayoría de las especies liquénicas tiene afinidad con el sustrato y aún sobre el sustrato de preferencia son muy específicos en su microhábitat, porque la estructura del bosque afecta a la distribución de los líquenes regulando el régimen de luz y humedad (Will Wolf et al. 2002). Se determinó la forma de crecimiento para cada especie liquénica, consignándose hábitos crustoso, folioso y fruticuloso.

El análisis de la vegetación liquénica se efectuó a partir de 90 inventarios fitosociológicos, 30 sobre troncos y 60 sobre ramas, utilizando el método de Braun Blanquet (1964). Los inventarios sobre tronco se realizaron en 10 forofitos de cada especie seleccionada, elegidos según el método del vecino más próximo (Pielou 1960). En cada uno de ellos se censaron dos ramas. Las muestras se obtuvieron de la cara sudsudoeste del tronco a 1,5 metros de la base. Para el análisis de presencia y cobertura de las especies se censó un rectángulo de $15 \mathrm{x}$ $20 \mathrm{~cm}$ en árboles de más de $10 \mathrm{~cm}$ de diámetro. En ramas de más de $15 \mathrm{~cm}$ de diámetro, ubicadas en la base del dosel, se realizó el inventario con el mismo cuadrado. Los muestreos se realizaron sobre las especies arbóreas A. quebracho-blanco, P. pugionata y Z. mistol.

Los datos fueron analizados con estadística descriptiva. Para la comparación de la diversidad entre las comunidades presentes en cada forofito se aplicó el índice de Shannon-Wiener (H') calculado según la siguiente fórmula: $\mathrm{H}^{\prime}=\sum \mathrm{p}_{\mathrm{i}} / \log \left(\mathrm{p}_{\mathrm{i}}\right)$, donde $\mathrm{p}$ es la proporción relativa (cobertura) de las i especies. Este índice presenta algunas limitaciones como: 1) supone que todas las especies es- tán incluidas en el muestreo, 2) se reduce la información de los datos originales y 3) es sensible a la variación del tamaño de la muestra. Sin embargo, es un índice muy usado y apropiado para pruebas estadísticas entre comunidades (Gilbert y Mejía 2002). Se organizó una matriz de especies por cobertura para aplicar una técnica de ordenación, el análisis de componentes principales (ACP) (Nimis 1984, Molina Moreno y Probanza 1992), con el fin de identificar grupos indicadores de comunidades.

\section{RESULTADOS}

Se encontró un total de 21 especies entre los tres forofitos (cuadro 1). La mayoría de las especies encontradas son foliosas, unas pocas fruticulosas y sólo una crustosa. No se determinaron especies crustosas presentes, debido a su dificultad y falta de claves. Como es común en los bosques, la forma de crecimiento predominante de los líquenes corticícolas fue la foliosa (cuadro 1). El sustrato más favorable para el desarrollo de líquenes fue Z. mistol, sobre el que se encontró el mayor número de especies, un total de 20 (cuadro 1), con cobertura homogéneamente distribuida en cada especie. Sobre $P$. pugionata se registraron 16 especies con cobertura total superior a los otros forofitos. El menor número de especies se presentó sobre A. quebracho-blanco con un total de 15 con la menor cobertura total. No se registran diferencias importantes entre las especies del tronco y las de rama.

La comunidad liquénica sobre $P$. pugionata tuvo como dominante a Rimelia reticulata (Taylor) Hale et Fletcher, con un valor de cobertura igual a la mitad del total para este forofito. Se presentó un grupo de especies acompañantes encabezadas por Physcia poncisnii Hue, Usnea densirostra Taylor y Canomaculina muelleri (Vain.) Elix et Hale. Sobre Z. mistol la especie dominante fue Punctelia microsticta (Müll. Arg.) Krog y las especies acompañantes fueron Heterodermia comosa (Eschw.) Follmann et Redon, Candelaria concolor (Dickson) Arnold, Usnea densirostra y Rimelia conferenda (Hale) Kurok. Sobre A. quebracho-blanco la especie dominante fue Rimelia reticulata y las acompañantes Canomaculina pilosa (Stizenb.) Elix et Hale, Parmotrema praesorediosum (Nyl.) Hale, Physcia erumpens Moberg y Physcia poncisnii Hue (cuadro 1).

El índice de Shannon para la cobertura liquénica fue de 0,90 para $P$. pugionata, de 1,04 para A. quebrachoblanco y de 1,19 para Z. mistol. La cobertura total fue, respectivamente, de $24 \%$, $45 \%$ y $70 \%$. Estos resultados muestran que los tres forofitos no representan tres comunidades bien diferenciadas, lo que es corroborado por el ACP (figura 1). El ordenamiento de las especies permite reconocer que en el eje 1 se destaca la dominancia de Rimelia reticulata, que fue la especie dominante en $A$. quebracho-blanco, mientras que en $P$. pugionata fue 
Cuadro 1. Media ( \pm desviación estándar) de cobertura de la comunidad liquénica sobre tres forofitos y formas de crecimiento de las especies de líquenes.

Mean values ( \pm standard deviation) of cover data in the lichen community on three phorophytes, and species growth forms.

\begin{tabular}{|c|c|c|c|c|}
\hline Especie de liquen & $\begin{array}{l}\text { Prosopis } \\
\text { pugionata }\end{array}$ & $\begin{array}{l}\text { Zizyphus } \\
\text { mistol }\end{array}$ & $\begin{array}{c}\text { Aspidosperma } \\
\text { quebracho-blanco }\end{array}$ & $\begin{array}{l}\text { Forma de } \\
\text { crecimiento }\end{array}$ \\
\hline Rimelia reticulata (Taylor) Hale et A. Fletcher & $5,30 \pm 8,49$ & - & $8,20 \pm 9,40$ & Folioso \\
\hline Punctelia microsticta (Müll. Arg.) Krog & $0,23 \pm 0,90$ & $3,90 \pm 5,56$ & $0,15 \pm 0,49$ & Folioso \\
\hline Heterodermia comosa (Eschw.) Follman et Redon & $0,23 \pm 0,41$ & $2,20 \pm 3,35$ & $0,30 \pm 0,92$ & Fruticuloso \\
\hline Parmotrema praesorediosum (Nyl.) Hale & - & $1,10 \pm 2,47$ & $2,00 \pm 4,72$ & Folioso \\
\hline Physcia ponsici Hue & $0,95 \pm 2,14$ & $0,05 \pm 0,22$ & $1,40 \pm 3,68$ & Folioso \\
\hline Physcia erumpens Moberg & $0,25 \pm 0,79$ & - & $1,40 \pm 2,91$ & Folioso \\
\hline Canomaculina pilosa (Stizenb.) Elix et Hale & $0,40 \pm 1,19$ & $0,10 \pm 0,45$ & $1,30 \pm 2,70$ & Folioso \\
\hline Rimeliella conferenda (Hale) Kurok. & $0,53 \pm 2,23$ & $1,15 \pm 2,50$ & $0,40 \pm 1,79$ & Folioso \\
\hline Punctelia hypoleucites (Nyl.) Krog & $0,35 \pm 1,18$ & $0,20 \pm 0,89$ & $0,95 \pm 2,37$ & Folioso \\
\hline Teloschistes cymbalyfer (Meyer) Müll. Arg. & $0,25 \pm 0,64$ & $0,85 \pm 2,18$ & $0,25 \pm 0,55$ & Fruticuloso \\
\hline Canomaculina muelleri (Vain.) Elix et Hale & $0,75 \pm 1,48$ & $0,55 \pm 1,10$ & - & Folioso \\
\hline Usnea densirostra Taylor. & $0,70 \pm 2,18$ & $1,00 \pm 4,47$ & - & Fruticuloso \\
\hline Myelochroa lindmanii (Lynge) Elix et Hale & - & $0,70 \pm 1,87$ & - & Folioso \\
\hline Candelaria concolor (Dickson) Arnold & $0,30 \pm 0,91$ & $0,70 \pm 1,22$ & - & Folioso \\
\hline Physcia rolfii Moberg & - & $0,65 \pm 1,84$ & $0,50 \pm 2,24$ & Folioso \\
\hline Ramalina celastri (Spreng.) Krog et Swinscow & $0,38 \pm 0,74$ & $0,60 \pm 1,27$ & $0,30 \pm 1,34$ & Fruticuloso \\
\hline Parmotrema austrosinense (Zahlbr.) Hale & $0,05 \pm 0,22$ & $0,30 \pm 0,73$ & $0,50 \pm 2,24$ & Folioso \\
\hline Hypotrachina livida (Taylor) Hale & $0,40 \pm 1,79$ & $0,30 \pm 1,34$ & - & Folioso \\
\hline Xanthoria sp. & $0,30 \pm 1,34$ & $0,15 \pm 0,49$ & - & Fruticuloso \\
\hline Physcia aipolia (Humb.) Fürnrohr & - & $0,15 \pm 0,49$ & $0,30 \pm 1,34$ & Folioso \\
\hline Lecanora sp. & - & $0,25 \pm 0,64$ & - & Crustoso \\
\hline
\end{tabular}

- : especie ausente.

excluyente y en Z. mistol ausente. En tanto, en el eje 2 se destacan dos especies con valores de cobertura importantes en Z. Mistol: P. microsticta y $U$. densirostra cuyos valores de cobertura son inferiores a los de $R$. reticulata. El resto de las especies se presenta en un grupo común que no muestra tendencia alguna.

\section{DISCUSIÓN Y CONCLUSIONES}

La comunidad liquénica presente en los tres forofitos estudiados es similar en cuanto al número y diversidad de especies. Las especies dominantes presentan una preferencia marcada por un sustrato, mientras las especies secundarias no tienen preferencia por uno u otro forofito. El número de especies es también similar al encontrado en el bosque serrano de la provincia de Córdoba (Estrabou y García 1995), con un total de 20 especies en los troncos de Lithraea ternifolia Barkley, especie dominante de este bosque. Esto muestra que la riqueza de especies en este ambiente semiárido es baja en general.

Aspidosperma quebracho-blanco y P. pugionata comparten la dominancia de una única especie, $R$. reticulata, que controla la energía de la comunidad liquénica. La segunda especie que controla la energía, sobre este forofito, es Parmotrema praesorediosum que está ausente en
$P$. pugionata, en el que aparece como acompañante $P$ hyscia poncisnii. En $Z$. mistol la ausencia de $R$. reticulata permite que Punctelia microsticta sea la dominante, secundada por $H$. comosa.

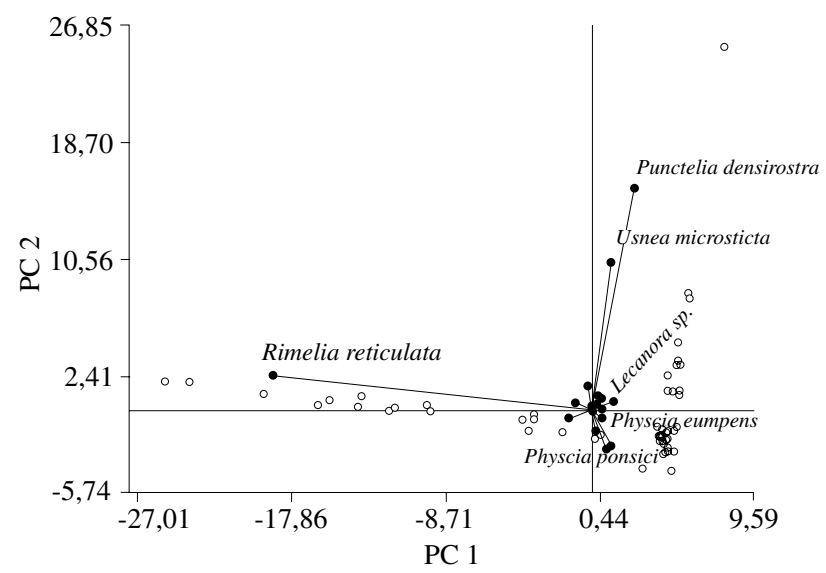

Figura 1. Análisis de especies sobre tres forofitos en el bosque chaqueño, Córdoba, Argentina. Ordenación de especies en el plano definido por los ejes 1 y 2 del ACP.

Lichens species analyses over three Chaco forest phorophytes in Córdoba, Argentina. Species ordination over PCA's axis 1 and axis 2 . 
Si se define una comunidad por sus dominantes y siguiendo a Braun Blanquet, sobre A. quebracho-blanco y $P$. pugionata hay un Reticulorum, mientras que sobre Z. mistol hay un Microstictorum. El resto de las especies aparece con valores más o menos representativos en los tres sustratos, sin definir una comunidad diferente para A. quebracho-blanco - P. pugionata y otra para Z. mistol.

Zizyphus mistol es un importante reservorio de especies ya que crecen prácticamente todas sobre él. El follaje de este forofito es muy abierto, lo que permite que la luz ingrese fácilmente. Como lo menciona Barkman (1958), este carácter en su estructura debe favorecer una mayor riqueza. En cambio, sobre $A$. quebracho-blanco y $P$. pugionata hay una comunidad con un dominante excluyente. En este sentido, la conservación de Z. mistol preservaría las especies liquénicas y una gran comunidad de insectos que se asocian a ellas (Valladares et al. 2006) en un bosque chaqueño con tendencia a la extinción ya que actualmente queda apenas un $10 \%$ de la superficie original.

El índice de Shannon no ha sido muy utilizado para explicar diversidad liquénica. En la comparación de la comunidad liquénica entre dos bosques en México, uno de los cuales recibe la contaminación de la capital, por su cercanía, y el otro se encuentra a $100 \mathrm{~km}$ de la misma, Zambrano et al. (2000) utilizan el índice de Shannon para comparar la diversidad. Tales autores encontraron menor diversidad en cercanías de la urbe $(1,6)$ y mayor en el bosque alejado $(2,1)$, y un total de 59 especies. Es decir, un ambiente más rico que el del presente estudio donde el índice de diversidad da valores similares. El manejo productivo de los ecosistemas de bosque en Córdoba, a pesar de estar regulado por normas de distinta jurisdicción, no ha sido exitoso en la preservación de especies y hábitats con interés de conservación. Se trata de un ambiente único que requiere urgentes medidas para su conservación. El presente estudio aporta a profundizar en la investigación de la estructura y funcionamiento de ecosistemas boscosos mediante el conocimiento del estado actual de los líquenes con el fin de colaborar en el desarrollo de planes de manejo en áreas boscosas naturales e intervenidas en la provincia de Córdoba. A partir de este trabajo se propone el mantenimiento de Z. mistol como soporte para la conservación del mayor número de especies liquénicas en este tipo de bosque. Asimismo, el monitoreo de las dos especies liquénicas dominantes, $R$. reticulata y $P$. microsticta, permitirá marcar cambios en la estructura del bosque. Se espera que estas especies indicadoras de procesos de cambio en el bosque señalen tempranamente cuándo y dónde el estado del bosque se está deteriorando.

\section{REFERENCIAS}

Barkman JJ. 1958. Phytosociology and ecology of criptogamic epiphytes. Van Gorcum, Assen. 628 p.

Braun Blanquet J. 1964. Planzensoziologie Grundzüge der Vegetationskunde. Springer. Vienne and New York. 864 p.

Cabido MR, MR Zak. 1999. Vegetación del norte de Córdoba. Secretaría de Agricultura, Ganadería y Recursos Renovables de la provincia de Córdoba y Agencia Córdoba Ambiente, Córdoba, Argentina. 56 p.

Cabrera AL. 1971. Fitogeografía de la República Argentina. Bol. Soc. Argent. Bot. 14(1-2): 1-42.

Elix JA. 1996. Biochemistry and secondary metabolites. In Nash TH. Lichen Biology. Cambridge University Press. p. $155-180$.

Estrabou C, L García. 1995. Comunidades liquénicas cortícolas sobre Lithraea ternifolia en las Sierras Chicas de la provincia de Córdoba, Argentina. Bot. Complut. 20:35-43.

Gilbert G, M Mejía eds. 2002. Manual para las Investigaciones de Biología de Campo. Instituto Smithsonian de Investigaciones Tropicales, Vicerrectoría de Inv. y Pos. Univ. de Panamá. 130 p.

Karlin UOT, LA Catalán, RO Coirini. 1994. El Chaco seco, un ambiente con vocación forestal. Facultad de Ciencias Agropecuarias. Universidad Nacional de Córdoba, Córdoba, Argentina. $160 \mathrm{p}$.

Llano GA. 1951. Economic uses of lichens. Ann. Rep. Smith. Inst. 385-422.

Molina Moreno JR, A Probanza. 1992. Pautas de distribución de biocenosis liquénicas epifíticas de un robledal de Somosierra (Madrid). Bot. Complut. 17: 65-78.

Nimis PL 1984. Phytosociology, ecology and phytogeography of epiphytic lichen vegetation in the Calamone Lake area (N-Apennines, Italy). Studia Geobot. 4: 1-109.

Pielou EC. 1960. A single mechanism to account for regular, random and aggregated populations. Journal of Ecology 48: $575-584$.

Prado DE. 1993. What is the Gran Chaco Vegetation in South America? I. A review. Contribution to the study of flora and vegetation of the Chaco. V. Candollea 48(1):145-172.

Valladares G, A Salvo, L Cagnolo. 2006. Habitat Fragmentation Effects on Trophic Processes of Insect-Plant Food Webs. Conservation Biology 20(1): 212.

Will Wolf S, PA Essen, P Neitlich. 2002. Monitoring Biodiversity and Ecosystem Function: Forests. In Nimis PL, C Scheidegger, PA Wolseley eds. Monitoring with lichens. Kluger Academic Publishers, Netherlands. p. 203-222.

Zak M, M Cabido y J G Hodgson. 2004. Do subtropical seasonal forests in the Grand Chaco, Argentina, have a future? Biological Conservation 120: 589-598.

Zambrano García A, TH Nash, MA Herrera Campos. 2000. Lichen decline in Desierto de los Leones (Mexico city). The Bryologist 103(3): 428-441. 